\begin{tabular}{|c|c|}
\hline $\begin{array}{l}\text { Cahiers } \\
\text { atı Recherche } \\
\text { Fonth Droits } \\
\text { Fondamentaux }\end{array}$ & $\begin{array}{l}\text { Cahiers de la recherche sur les droits } \\
\text { fondamentaux }\end{array}$ \\
\hline & $\begin{array}{l}5 \mid 2006 \\
\text { L'enfant }\end{array}$ \\
\hline
\end{tabular}

\title{
Travaux des membres du CRDFED
}

\section{(2) OpenEdition}

1 Journals

Édition électronique

URL : https://journals.openedition.org/crdf/7222

DOI : $10.4000 /$ crdf.7222

ISSN : 2264-1246

Éditeur

Presses universitaires de Caen

Édition imprimée

Date de publication : 31 décembre 2006

Pagination : 125-126

ISBN : 978-2-84133-277-9

ISSN : $1634-8842$

Référence électronique

"Travaux des membres du CRDFED », Cahiers de la recherche sur les droits fondamentaux [En ligne], 5 | 2006, mis en ligne le 15 décembre 2020, consulté le 16 novembre 2022. URL : http://

journals.openedition.org/crdf/7222 ; DOI : https://doi.org/10.4000/crdf.7222 


\section{Travaux des membres du CRDFED}

\section{Autour des droits fondamentaux}

\section{A. Articles et communications}

AKAndJI-Kombé Jean-François, «The material impact of the "jurisprudence" of the European Committee of Social Rights", in Social Rights in Europe, G. De Burca (dir.), Oxford, Oxford University Press, 2005.

Akandji-Kombé Jean-François, "Accord de Cotonou ", in P. Tavernier et C. Heyns, Recueil juridique des droits de l'homme en Afrique, Bruxelles, Bruylant, 2004.

AKANDJI-KombÉ Jean-François, «Avant-propos sur l'inscription des droits sociaux dans la Convention européenne des droits de l'homme. Préalables méthodologiques sur la justiciabilité des doits sociaux", Cahiers de la recherche sur les droits fondamentaux, $\mathrm{n}^{\circ}$ 3, 2004, p. 83-92.

Armand Gilles, «La dignité des malades en fin de vie », in $L a$ Portée de l'article 3 de la Convention européenne des droits de l'homme (Actes du colloque de Caen, 12-13 mai 2005), C.-A. Chassin (dir.), Bruxelles, Bruylant (Rencontres européennes), 2006, p. 181-208.

ARmand Gilles, «Que reste-t-il de la protection constitutionnelle de la liberté individuelle?", Revue française de droit constitutionnel, $\mathrm{n}^{\circ} 65,2006$, p. 37-72.

Armand Gilles, «L'ordre public de protection individuelle», Revue de la recherche juridique, Droit prospectif, 2004/3, p. 1583-1646.

Armand Gilles, Fouquet-Armand Maud, «L'uniformité territoriale dans la jouissance et l'exercice des droits et libertés fondamentaux en France ", Cahiers de la recherche sur les droits fondamentaux, $\mathrm{n}^{\circ}$ 2, 2003, p. 11-32.

Chassin Catherine-Amélie, "L'article 3 de la Convention européenne des droits de l'homme, instrument de protection des mineurs", in La Portée de l'article 3 de la Convention européenne des droits de l'homme (Actes du colloque de Caen, 12-13 mai 2005), C.-A. Chassin (dir.), Bruxelles, Bruylant (Rencontres européennes), 2006, p. 235-261.

Chassin Catherine-Amélie, «Asile», Répertoire Dalloz de droit international, septembre 2005.

Chassin Catherine-Amélie, «Panorama français du droit de l'apatridie ", $R F D A, \mathrm{n}^{\circ}$ 2, 2003, p. 324-330.

FonTAINE Lauréline, «Le pluralisme comme théorie des normes ", in Le Pluralisme (Actes du colloque de Caen, novembre 2006), L. Fontaine (dir.), à paraître aux éditions Bruylant.

Fontaine Lauréline, Conclusions de la journée d'études sur La Citoyenneté européenne (Actes des $4^{\mathrm{e}}$ Rencontres européennes de Caen, 26 novembre 2004), S. Leclerc, J.-F. AkandjiKombé (dir.), Bruxelles, Bruylant (Rencontres européennes), à paraître.
Fontaine Lauréline, «Les sources nouvelles du droit de l'environnement», contribution au colloque Droit et Environnement organisé par l'Association Henri Capitant et préparé par François-Guy Trébulle à l'Université de Caen le 6 avril 2006, à paraître.

LARralde Jean-Manuel, «L'article 3 CEDH et les personnes privées de liberté ", in La Portée de l'article 3 de la Convention européenne des droits de l'homme (Actes du colloque de Caen, 12-13 mai 2005), C.-A. Chassin (dir.), Bruxelles, Bruylant (Rencontres européennes), 2006, p. 209-234.

LARRALDE Jean-Manuel, «Les réponses du droit international à la question des enfants soldats ", Cahiers de la recherche sur les droits fondamentaux, $\mathrm{n}^{\circ}$ 5, 2006, p. 65-78.

Larralde Jean-Manuel, «La Convention européenne des droits de l'homme et la protection de l'identité sexuelle», Revue trimestrielle des droits de l'homme, $\mathrm{n}^{\mathrm{0}}$ 65, 2006, p. 35-54.

LARRALDE Jean-Manuel, « La protection des religions minoritaires en droit international et européen ", Cahiers de la recherche sur les droits fondamentaux, $\mathrm{n}^{\circ}$ 4, 2005, p. 157-168.

LARRALDE Jean-Manuel, « La protection du détenu par l'action du Comité européen pour la prévention de la torture ", Cahiers de la recherche sur les droits fondamentaux, $\mathrm{n}^{\circ} 3,2004$, p. 29-42.

LARRALDE Jean-Manuel, «Les langues régionales en droit français", in Europäische Sprachenpolitik / European Language Policy (Actes du colloque international de Würzburg, 6-8 juin 2002), R. Ahrens (dir.), Heidelberg, Universitätsverlag Winter, 2003, p. 191-214.

LARRALDe Jean-Manuel, «La Convention européenne des droits de l'homme et la protection de groupes particuliers ", Revue trimestrielle des droits de l'homme, $\mathrm{n}^{\circ}$ 56, 2003, p. 12471275 .

LARRALDE Jean-Manuel, «Les droits des personnes incarcérées: entre punition et réhabilitation ", Cahiers de la recherche sur les droits fondamentaux, $\mathrm{n}^{\circ}$ 2, 2003, p. 63-76.

LECLERC Stéphane, «Les droits du citoyen européen », in $\mathrm{La}$ Citoyenneté européenne (Actes des $4{ }^{\mathrm{e}}$ Rencontres européennes de Caen, 26 novembre 2004), S. Leclerc, J.-F. AkandjiKombé (dir.), Bruxelles, Bruylant (Rencontres européennes), à paraître.

Le Grand Vincent, «La liberté du cinéma à l'épreuve de l'objectif de protection des mineurs » - Commentaire de l'arrêt Conseil d'État, 4 février 2004, "Association Promouvoir» publié dans le numéro 2004/ 4 de la Revue de la recherche juridique, p. 2647-2664.

Redor-Fichot Marie-Joëlle, «Universalisme et pluralisme », in Le Pluralisme (Actes du colloque de Caen, novembre 2006), L. Fontaine (dir.), à paraître aux éditions Bruylant. 
Redor-Fichoт Marie-Joëlle, «Rapport introductif», in $\mathrm{La}$ Citoyenneté européenne (Actes des 4 Rencontres européennes de Caen, 26 novembre 2004), S. Leclerc, J.-F. Akandji-Kombé (dir.), Bruxelles, Bruylant (Rencontres européennes), à paraître.

Redor-Fichot Marie-Joëlle, «L'article 3 de la Convention européenne des droits de l'homme dans la jurisprudence administrative ", in L'Article 3 de la Convention européenne de sauvegarde des droits de l'homme et des libertés fondamentales, C.-A. Chassin (dir.), à paraître aux éditions Bruylant.

Redor-Fichoт Marie-Joëlle, "Laïcité et principe de nondiscrimination ", Cahiers de la recherche sur les droits fondamentaux, $\mathrm{n}^{\circ} 4$ décembre 2005, p. 87-98.

Redor-Fichot Marie-Joëlle, "Synthèse du colloque Surveiller et punir/Surveiller ou punir? Perspectives de la peine privative de liberté», Cahiers de la recherche sur les droits fondamentaux, $\mathrm{n}^{\circ} 3$, 2004, p. 77-80.

\section{B. Ouvrages}

AkandjI-Kombé Jean-François, Les Obligations positives en vertu de la Convention européenne des droits de l'Homme, Strasbourg, Éditions du Conseil de l'Europe (Précis sur les droits de l'homme, $\mathrm{n}^{\circ}$ 7), 2006

Akandji-Kombé Jean-François, Leclerc Stéphane (dir.), La Citoyenneté européenne (Actes des 4 e Rencontres européennes de Caen, 26 novembre 2004), Bruxelles, Bruylant (Rencontres européennes), à paraittre.

Chassin Catherine-Amélie (dir.), La Portée de l'article 3 de la Convention européenne des droits de l'homme (Actes du colloque de Caen, 12-13 mai 2005), Bruxelles, Bruylant (Rencontres européennes), 2006.

Fontaine Lauréline (dir.), Le Pluralisme (Actes du colloque de Caen, novembre 2006), à paraître aux éditions Bruylant.

\section{Autour des évolutions du droit}

\section{A. Articles et communications}

Castillo Maria, «Les grandes étapes de l'évolution de l'ordre économique international ", in Dictionnaire de droit international économique, $\mathrm{O}$. Audéoud, $\mathrm{H}$. Ghérari (dir.), à paraitre aux éditions Pedone.

Lajoye Christophe, «Patrimoine des collectivités territoriales ", JurisClasseur administratif, fasc. 128/2005.

Lajoye Christophe, «Le critère de l'aménagement spécial dans l'acception du domaine public ", Droit et ville, $\mathrm{n}^{\circ} 56 /$ 2004, p. 138 sq.

Lajoye Christophe, «Organes de l'achat public», JurisClasseur: contrats et marchés publics, fasc. 60-10, 2004

LAMUlLE Thierry, «Citoyenneté européenne et fiscalité », in La Citoyenneté européenne (Actes des $4^{\mathrm{e}}$ Rencontres européennes de Caen, 26 novembre 2004), S. Leclerc, J.-F. AkandjiKombé (dir.), Bruxelles, Bruylant (Rencontres européennes), à paraître.
Lamulle Thierry, "Les redevances et les produits domaniaux", intervention au colloque de Lille (novembre 2006) consacré au Code général de la propriété des personnes publiques, à paraître.

LARRALDE Jean-Manuel, «L'acte II de la décentralisation : la France en marche vers l'État régional ? ", Politeia, $n^{\circ} 6$, automne 2004, p. 233-254.

Leclerc Stéphane, «Les régions et les Fonds de la cohésion économique et sociale", in L'Union européenne, L. Dubouis (éd.), Paris, La Documentation française (Les notices), 2004.

LECLERC Stéphane, «L'évolution du cadre juridique et conceptuel de la cohésion économique et sociale : la nouvelle réglementation des Fonds structurels (2000-2006)", in L'Europe et les régions: quinze ans de cohésion économique et sociale (Actes des $3^{\mathrm{e}}$ Rencontres européennes de Caen, 14-15 mars 2002), S. Leclerc (dir.), Bruxelles, Bruylant (Rencontres européennes), 2004, p. 71-89.

LECLERC Stéphane, «Les facteurs de convergence de la démocratie locale et régionale dans l'Union européenne », in Démocratie et Management local (Actes des $5{ }^{\mathrm{e}}$ Rencontres VilleManagement, Bayonne, 6-7 septembre 2001), R. Le Duff, J.-J. Rigal (dir.), Paris, Dalloz (Thèmes et commentaires), 2003.

Le Grand Vincent, «Léon Blum : un juriste au parlement », communication au colloque intitulé Les juristes et la loi, organisé au Sénat le 5 mai 2006 par le Comité d'histoire politique et parlementaire (CHPP) et le Centre d'études constitutionnelles et politiques (CECP - Université Paris II), à paraître dans un numéro hors-série de la revue Parlement(s).

Le Grand Vincent, «Léon Blum (1872-1950): une lecture républicaine du parlementarisme", in Les Juristes face au politique: le droit, la gauche, la doctrine sous la Troisième République, Paris, Kimé, 2003, p. 123-146.

\section{B. Ouvrages}

Akandji-Kombé Jean-François, Fenet Alain, LamblinGourdin Anne-Sophie, Mondielli Éric et ProutièreMaulion Gwenaëlle, Droit des relations extérieures de l'Union européenne, Paris, Litec, 2007.

Lajoye Christophe, Droit des marchés publics, $2^{\mathrm{e}}$ éd., Paris, Gualino, 2005.

Lamulle Thierry, Mémento droit fiscal, Paris, Gualino, édition annuelle.

LAmulle Thierry, Manuel de droit fiscal, Paris, Gualino, 2005.

LECLERC Stéphane, L'Essentiel du droit institutionnel de l'Union européenne, Paris, Gualino (Les essentiels), 2006.

Leclerc Stéphane, Mémento - Les institutions de l'Union européenne, Paris, Gualino (Mémentos LMD), 2005.

LeClerc Stéphane, Mémento - Droit institutionnel de l'Union et des Communautés européennes, Paris, Gualino (Fac-Universités), 2003.

Thouroude Jean-Jacques, La Responsabilité du fait des ouvrages publics communaux, à paraître aux éditions du Moniteur. 